\title{
Influence of Cellulose on the Mechanical and Thermal Stability of ABS Plastic Composites
}

\author{
K. Crews, ${ }^{1}$ C. Huntley, ${ }^{1}$ D. Cooley, ${ }^{1}$ B. Phillips, ${ }^{1}$ and M. Curry ${ }^{1,2}$ \\ ${ }^{1}$ Department of Materials Science and Engineering, Tuskegee University, Tuskegee, AL 36088, USA \\ ${ }^{2}$ Department of Chemistry, Tuskegee University, Tuskegee, AL 36088, USA \\ Correspondence should be addressed to M. Curry; currym@mytu.tuskegee.edu
}

Received 15 January 2016; Revised 30 March 2016; Accepted 3 May 2016

Academic Editor: Quanling Yang

Copyright (c) $2016 \mathrm{~K}$. Crews et al. This is an open access article distributed under the Creative Commons Attribution License, which permits unrestricted use, distribution, and reproduction in any medium, provided the original work is properly cited.

\begin{abstract}
Microcrystalline cellulose was explored as possible biodegradable fillers in the fabrication of ABS plastic composites. TGA indicates that upon inclusion of cellulose microcrystals the thermal stability of the ABS plastics was improved significantly when compared to the neat ABS plastic counterparts. Furthermore, inclusion of extracted cellulose from plant biomass showed a higher thermal stability with maximum decomposition temperatures around $131.95^{\circ} \mathrm{C}$ and $124.19^{\circ} \mathrm{C}$ for cellulose from cotton and Hibiscus sabdariffa, respectively, when compared to that of the purchased cellulose. In addition, TMA revealed that the average CTE value for the neat ABS and 1:1 ratio of cellulose to ABS fabricated in this study was significantly lower than the reported CTE (ca. $\left.73.8 \mu \mathrm{m} / \mathrm{m}^{\circ} \mathrm{C}\right)$.
\end{abstract}

\section{Introduction}

Due to the rapid growth of waste from electrical and electronic equipment, a considerable amount of focus has been placed on exploring new sources that will allow the creation of unique polymeric materials that can be used in the development of biobased composites. Today, there are two categories of biodegradable polymers: synthetic and natural polymers. Though not all synthetic polymers portray such nature, upon the incorporation of additives these polymers mimic biodegradable behavior. Vroman and Tighzert [1] reported that natural polymers generally offer fewer advantages than synthetic polymers; however, natural polymers offer the key advantage of biodegradability. That being said, producing these composites with biodegradable nanomaterials will give rise to inexpensive, natural, renewable materials with little to no negative impacts on the environment.

Cellulose has been used to combat the issues of sustainability [2] and is a common biopolymer that is being used widely [3-8]. Cellulose is a complex carbohydrate made up of several thousand glucose molecules linked end to end, and due to its intra- and intermolecular hydrogen bonding various ordered crystalline arrangements are observed. Given the abundance of natural resources available for cellulose and the unique mechanical properties of highly crystalline cellulose (micro- or nanocrystalline cellulose), it has great potential for use as fillers to increase composite material properties in comparison to unfilled polymer matrix counterparts. That is, cellulose composites have the potential to be light-weight, low-cost, and environmentally friendly with improved thermal and mechanical properties. Due to the incompatibility between generally hydrophobic host polymer matrix and hydrophilic natural fiber, combined with lower thermal resistance of the cellulosic material, most cellulose research has primarily focused on its extraction from different plants modification and its use in the reinforcement of various polymer matrices [9-16]. Common techniques for composites fabrication include but are not limited to casting, templating $[6,17,18]$, and compounding or extrusion $[8,19]$.

Currently, acrylonitrile-butadiene-styrene (ABS) is one of the major components used in the fabrication of plastics casings for computers, monitors, keyboards, and other similar components. A life cycle assessment conducted in our group, but not discussed in this paper, has shown that the thermal processing of ABS has an impact on several environmental categories, having the greatest impact on 


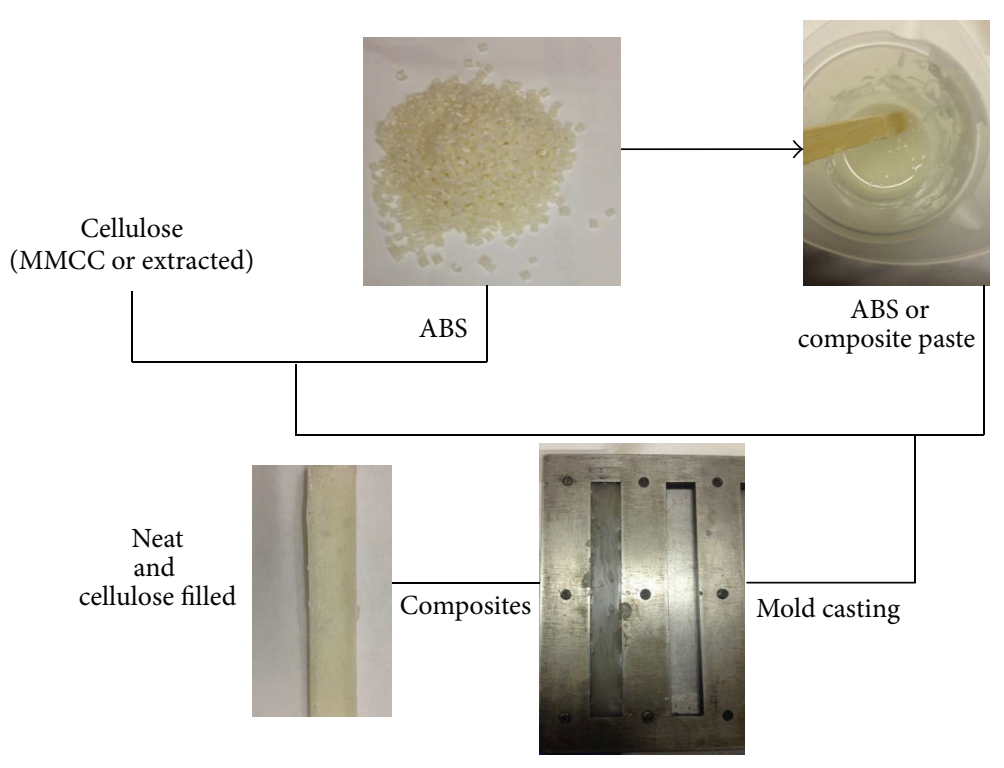

FIGURE 1: A typical flowchart depicting the method used to fabricate neat and cellulose-based ABS plastic composites for comparative analyses.

global warming. Though the monomers (acrylonitrile, butadiene, and styrene) of ABS may not be extremely harmful, this terpolymer possesses different properties and produces volatile organic compounds (VOCs) during pyrolysis, combustion, and extrusion [20, 21]. The list of possible VOCs includes hydrocarbons, aromatic hydrocarbons, alcohols, ketones, aldehydes, acids, and acrylonitrile. To conform to more biofriendly composites, micron-sized cellulose, a natural biopolymer, is used as filler for an acrylonitrilebutadiene-styrene (ABS) matrix. There are several challenges within cellulose particles, such as the capability to absorb moisture, but they have promising properties as a biofiller for polymers. Cellulose fillers have the potential of enhancing the composite properties (e.g., thermal or mechanical) of plastics formed using ABS, as well as increasing its biodegradation by organisms and recycling methods [11]. Hence, in this report, polymer dissolution and mold cast techniques were used for the fabrication of cellulose-based ABS composites as an alternative to a thermal process due to the volatile organic compounds (VOCs) released during thermal processing (i.e., recycling or extrusion).

\section{Materials and Methods}

2.1. Materials. Dichloromethane $\geq 99.5 \%$ ACS reagent was purchased from Sigma-Aldrich while the Lustran ABS 552 (ABS) was purchased from PolyOne Corporation. The manufactured microcrystalline cellulose (MMCC) was received from CreaFill Fibers Corp. as TC40 CreaTech. For the extraction of cellulose, acetic acid, acetone, sodium chlorite, sodium hydroxide, and sulfuric acid, ACS reagent grade chemicals were purchased from Sigma-Aldrich and used as received. Hibiscus sabdariffa was obtained from the local farm. The cotton was purchased from the local convenience store.
2.2. Sample Preparation. Cellulose extracted from Hibiscus and cotton was carried out using the experimental procedures as described by Huntley et al. [17]. In short, solutions of acetic acid (90 wt $\%)$, sodium chlorite ( $9 \mathrm{wt} \%)$, a buffer solution, and sulfuric acid (32 vol\%) were prepared for cellulose extraction. Acetic acid was used for a pretreatment process followed by bleaching. The resulting product was then hydrolyzed using sulfuric acid. Following hydrolysis, centrifugation and sonication were performed for the complete extraction of crystalline cellulose. The flow chart in Figure 1 depicts the method utilized to fabricate neat ABS, as well as composites reinforced with MMCC and extracted celluloses. This method has been found to be beneficial due to the method being carried out at room temperature, which increases the cost effectiveness during production [18]. The ABS pellets and cellulose were added to a plastic mixing cup in varying ratios; see Table 2. As-received dichloromethane was added to dissolve the ABS pellets. Utilizing a stir stick, a manual mixing method was used. The ABS and cellulose ratios were mixed for several minutes to achieve uniform distribution of the cellulose fibers. The resulting paste was poured in a metal flexure mold and allowed to dry at room temperature for 48 hours or longer [22]. (Cellulose dispersion studies need to be conducted due to a lack in mechanical mixing procedures to ensure homogeneous mixing of the materials.)

2.3. X-Ray Diffraction (XRD). XRD analyses were performed using a Rigaku D/MAX 2200 X-ray diffractometer using $\mathrm{CuK} \alpha$ radiation with wavelength $\lambda=1.54 \AA$ and $40 \mathrm{kV}$ and $30 \mathrm{~A}$. Analyses of dried celluloses were performed on a glass slide from $0^{\circ}$ to $50^{\circ}$ of $2 \theta$ angle at a rate of $5^{\circ} \mathrm{C} / \mathrm{min}$. Proceeding data collection, the results were characterized utilizing PDF data base of Joint Committee on Powder Diffraction Standards (JCPDS) along with literature findings. 


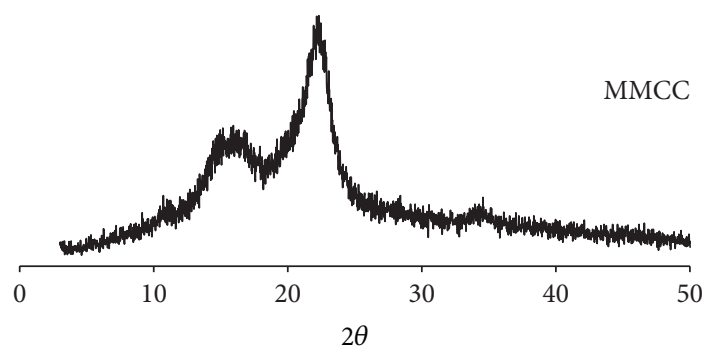

(a)

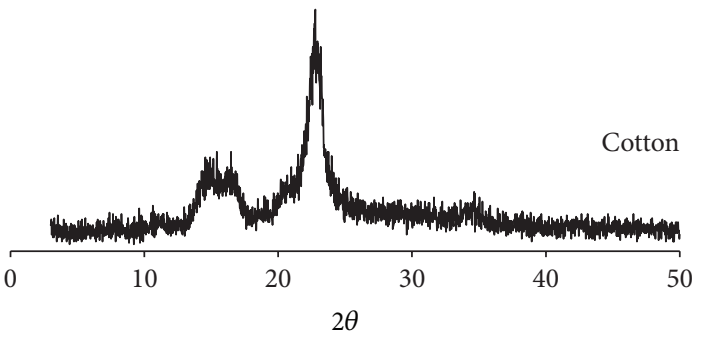

(b)

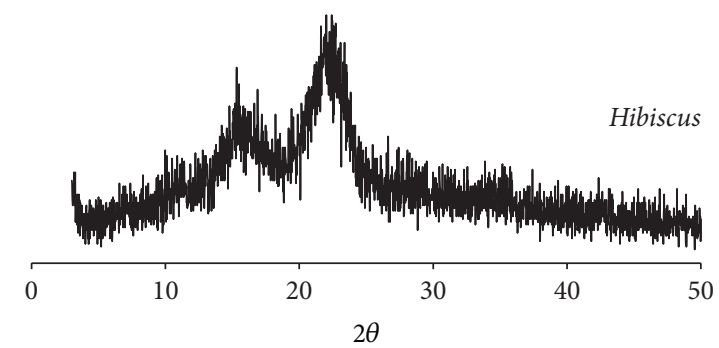

(c)

FIGURE 2: XRD spectra revealing the CI structure for purchased MMCC (a) and cellulose extracted from cotton and Hibiscus (b) and (c), respectively, via strong acid hydrolysis.

2.4. Scanning Electron Microscopy (SEM). SEM images were collected from a Zeiss EVO 50 VP SEM operating at an acceleration voltage of $3 \mathrm{keV}$ for the purchased cellulose and $20 \mathrm{keV}$ for the extractions. Additionally, gold sputter coating was achieved on an EMS 550X sputter coating device.

\subsection{Modulated Differential Scanning Calorimetry (MDSC).} Thermal analysis was carried out using custom MDSC heat only, with sample sizes remaining in a range of $10-13 \mathrm{mg}$. These analyses, for the dried celluloses along with the composites and pellets, were carried out on TA Instrument DSC Q2000 equipped with aluminum hermetic pans. The samples were analyzed under nitrogen gas at $50 \mathrm{~mL} / \mathrm{min}$. While the cellulose and pellet samples were ramped to $400^{\circ} \mathrm{C}$ and $300^{\circ} \mathrm{C}$ at a rate of $5^{\circ} \mathrm{C} / \mathrm{min}$, the composites were subjected to a different method to determine the glass transition temperature $\left(T_{g}\right)$ after thermal stresses were relieved. The ABS composite samples were ramped from $30^{\circ} \mathrm{C}$ to $140^{\circ} \mathrm{C}$, cooled to $30^{\circ} \mathrm{C}$, and ramped again to $150^{\circ} \mathrm{C}$.

2.6. Thermogravimetric Analysis (TGA). TGA analyses were performed using a TA Instrument TGA Q500 under a nitrogen atmosphere. The mass of all samples ranged from 13 to $15 \mathrm{mg}$ and they were tested using pretared platinum TGA pans. The samples were purged with nitrogen at a rate of $40 \mathrm{~mL} / \mathrm{min}$. After drying the extracted cellulose samples, these samples along with the purchased cellulose were analyzed at a rate of $5^{\circ} \mathrm{C} / \mathrm{min}$ from $30^{\circ} \mathrm{C}$ to $400^{\circ} \mathrm{C}$. On the other hand, the composites samples were analyzed at an identical rate with a ramping range of $30^{\circ} \mathrm{C}$ to $300^{\circ} \mathrm{C}$.

2.7. Thermomechanical Analysis (TMA). TMA were completed on TA Instrument TMA Q400. The samples were analyzed at $5^{\circ} \mathrm{C} / \mathrm{min}$ at $30^{\circ} \mathrm{C}$ to $90^{\circ} \mathrm{C}$ under thermal expansion mode, with a force of $0.3 \mathrm{~N}$ in a nitrogen atmosphere.

\section{Results and Discussions}

3.1. XRD Analysis of the Manufactured and Extracted Microcrystalline Cellulose. Figures 2(a)-2(c) display the crystalline structure for MMCC and cellulose extracted from cotton and Hibiscus. In Figure 2(a), XRD analysis verifies cellulose I (CI) polymorph structure. As can be observed, the existence of peaks with $2 \theta$ values of $15^{\circ}, 22^{\circ}$, and $35^{\circ}$ is shown. These results match well with previously reported results on the structure of native cellulose [2]. In addition, in previously reported literature for different biomass sources by Contos et al. [21], Ford et al. [23], and Kargarzadeh et al. [24], similar structural arrangements of the extracted cellulose were observed when analyzing by XRD.

Likewise, the extracted celluloses featured in Figures 2(b) and 2(c) exhibit similar XRD behavior patterns to that shown in Figure 2(a). This report is made evident due to the corresponding $2 \theta$ values of $15^{\circ}, 22^{\circ}$, and $35^{\circ}$. For the extracted cotton spectra, the peak at $15^{\circ}$ portrays two peaks overlapping which is usually observed in a $\mathrm{CI} \alpha / \mathrm{CI} \beta$ mixed XRD pattern as well as mercerized cellulose that has not been fully converted to cellulose II [23, 25]. O'Sullivan [26] reports that native cellulose, cellulose I, only exists in a mixture ( $\mathrm{I} \alpha$ and $\mathrm{I} \beta$ ). A true conversion of $\mathrm{CI}$ to $\mathrm{CII}$ occurs through mercerization $[26,27]$ (swelling due to sodium hydroxide) or regeneration (solvent solubilization and reprecipitation by water dilution [28]). Meanwhile, the peak at $22^{\circ}$ is more defined in comparison to the MMCC spectra which we attribute to the smaller diameter fibers and increased shape uniformity of the cotton fibers versus its MMCC and Hibiscus 


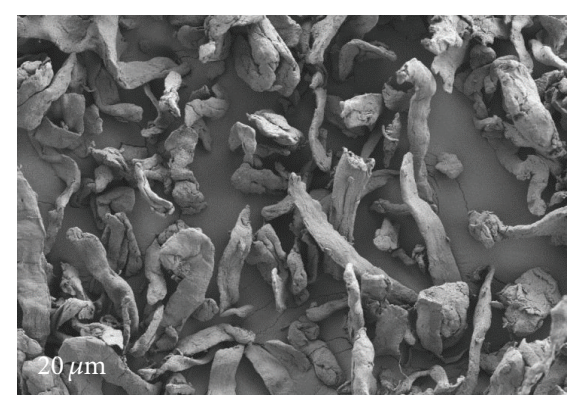

(a)

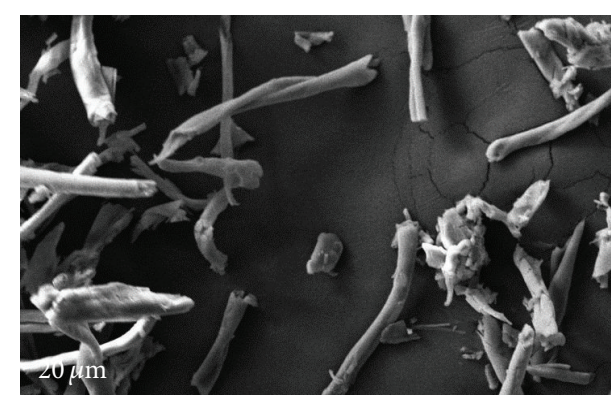

(b)

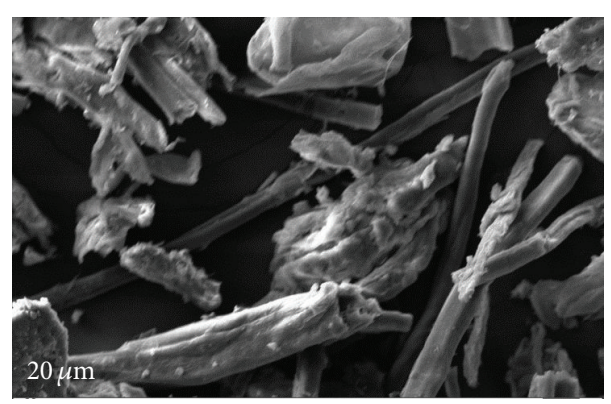

(c)

FIGURE 3: Scanning electron micrographs revealing the shape and size for purchased cellulose, MMCC (a), and cellulose extracted from cotton (b) and Hibiscus (c).

counterparts; see Figures 3(a)-3(c). In addition, based on the peak height XRD method reported in the literature [28] for calculation of cellulose crystallinity, we have determine that the cellulose extracted from cotton has the highest crystallinity (ca. $88.46 \%$ ) based on our method of extraction. Moon et al. [2] and Kargarzadeh et al. [24] reported similar values for the microcrystalline cellulose dimensions [2] and crystallinity $[2,24]$.

3.2. SEM Analysis of the Manufactured and Extracted Microcrystalline Cellulose. Scanning electron micrographs are presented in Figures 3(a)-3(c) revealing the size and shape of MMCC and extracted celluloses. Figure 3(a) shows that the MMCC crystals are rod-like fibers with diameters and lengths in the micron-size range. These cellulose fibers appear to be free of agglomeration and not uniform in length; that is, longer and shorter length scale fibers are observed. However, the cotton extracted cellulose counterpart featured in Figure 3(b) apparently exhibits similar fiber-like crystals with increased uniformity in its shape when compared to the manufactured microcrystalline cellulose in Figure 3(a). Furthermore, the length of the cotton extracted cellulose appears longer than that of the MMCC with lengths of approximately $100 \mu \mathrm{m}$ and $200 \mu \mathrm{m}$, respectively.

Similar results are observed for cellulose extracted from Hibiscus; see Figure 3(c). Hibiscus extracted cellulose exhibits rod-like fibers with lengths and diameters on the micrometer scale. Furthermore, large scale aggregation of cellulose fibers is observed for Hibiscus extracted cellulose, which is attributed to the larger fiber sizes $(\geq 300 \mu \mathrm{m})$ observed when compared to its cotton extracted or purchased cellulose counterparts. It has been reported that, depending on the mechanistic nature of the drying process and extraction methodology used, induced large scale aggregation of cellulose fibers will be observed $[6,19,29]$. Thus, based on reports from the literature and previous findings reported by our group $[17,25]$, we propose here that a similar mechanism of induced aggregation is occurring due to a combination of both processing and drying methodologies.

\subsection{Thermal Analysis of the Manufactured and Extracted} Microcrystalline Celluloses. Figure 4 reveals the DSC curve showing the thermal behavior of MMCC. This analysis reveals exothermic as well as endothermic peaks corresponding to water vaporization, degradation, char formation, and oxidation $\left(90-150^{\circ} \mathrm{C}, 262^{\circ} \mathrm{C}, 275^{\circ} \mathrm{C}\right.$, and $337^{\circ} \mathrm{C}$, resp.). Similar results were observed by Sinha and Rout [30]. Here it is reported that water vaporization appears as a broad endothermic peak in the range of $60-140^{\circ} \mathrm{C}$, another small endothermic peak present is indicative of the thermal degradation of hemicelluloses and the glycosidic linkages of cellulose at $290^{\circ} \mathrm{C}$, and further decomposition of cellulose occurs around $365^{\circ} \mathrm{C}$ causing char formation. The char is later oxidized along with the remaining mass being consumed, around $431^{\circ} \mathrm{C}$. As can be seen, similar results are observed in Figure 4 with a broad endothermic peak appearing with a range between 90 and $150^{\circ} \mathrm{C}$ but centered at around $115^{\circ} \mathrm{C}$ and corresponding small endothermic and exothermic peaks, $262^{\circ} \mathrm{C}, 275^{\circ} \mathrm{C}$, and $337^{\circ} \mathrm{C}$, respectively, indicating hemicellulose and glycosidic linkages decomposition, continuing with further cellulose degradation causing char formation, followed by the oxidation of char. In addition, temperature 
TABLE 1: Tabulated DSC and TGA values for both purchased and extracted cellulose.

\begin{tabular}{lccccc}
\hline \multirow{2}{*}{ Name } & DSC & \multicolumn{3}{c}{ TGA } \\
& $T_{g}\left({ }^{\circ} \mathrm{C}\right)$ & Residue $(\%)$ & Onset $\left({ }^{\circ} \mathrm{C}\right)$ & Rate $\left(\% /{ }^{\circ} \mathrm{C}\right)$ & $\mathrm{Max} \operatorname{decomp}\left({ }^{\circ} \mathrm{C}\right)$ \\
\hline MMCC & N/A & $14.01 \pm 0.22$ & $257.88 \pm 8.62$ & $1.75 \pm 0.07$ & $333.37 \pm 0.61$ \\
Hibiscus & N/A & $28.68 \pm 0.91$ & $165.82 \pm 2.87$ & $0.57 \pm 0.09$ & $252.08 \pm 5.64$ \\
Cotton & N/A & $32.97 \pm 0.15$ & $131.90 \pm 2.83$ & $0.36 \pm 0.00$ & $207.31 \pm 2.53$ \\
\hline
\end{tabular}

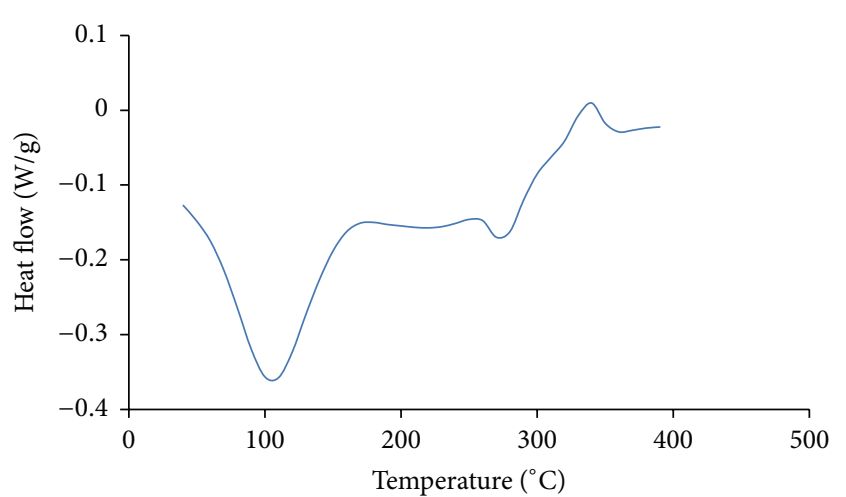

FIGURE 4: DSC curve showing the thermal behavior of MMCC.

ranging between 200 and $250^{\circ} \mathrm{C}$ apparently reveals a high thermal stability region for the MCC. That is, once the absorbed water has been driven off, the cellulose structure exhibits high resistance to degradation between $200^{\circ} \mathrm{C}$ and $250^{\circ} \mathrm{C}$.

The thermogravimetric analysis, TGA, of the MMCC and extracted celluloses can be observed in Figure 5 while the corresponding tabulated values that were obtained from both TGA and differential scanning calorimetry, DSC, analyses are provided in Table 1. The onset of degradation for the manufactured cellulose is higher than that of the extracted cellulose, with an approximate value of $258^{\circ} \mathrm{C}$; see Table 1 . The maximum decomposition temperature for the MMCC was observed at $333^{\circ} \mathrm{C}$ at a rate of $1.75 \% /{ }^{\circ} \mathrm{C}$. However, the TGA for the extracted celluloses exhibited significantly lower onset and maximum decomposition values (i.e., Hibiscus and cotton, $166^{\circ} \mathrm{C}$ and $132^{\circ} \mathrm{C}$ and $252^{\circ} \mathrm{C}$ and $207^{\circ} \mathrm{C}$, resp.) when compared to their manufactured counterpart. We attribute the significant difference in TGA values exhibited for the comparison of manufactured and extracted cellulose to the extraction methodology. That being said, the Hibiscus and cotton exhibited similar rates of mass loss at the maximum decomposition values when compared. Similar results have been observed for cellulose extracted from Hibiscus cannabinus (kenaf bast fibers). Kargarzadeh et al. [24] reported a sharp weight loss at $300^{\circ} \mathrm{C}$ for unhydrolyzed cellulose, which can be observed in Figure 4 for the MMCC due to its undergoing the pulping process which does not utilize hydrolysis [31]. According to the manufacturers, MMCC was produced by micronization of hardwood pulp. Kargarzadeh et al. [24] also reported that longer hydrolysis time makes for shorter fibers. Huntley et al. [25] reported that acids used during hydrolysis affect the thermal stability of extracted celluloses. The functional groups attached to the active sites on cellulose

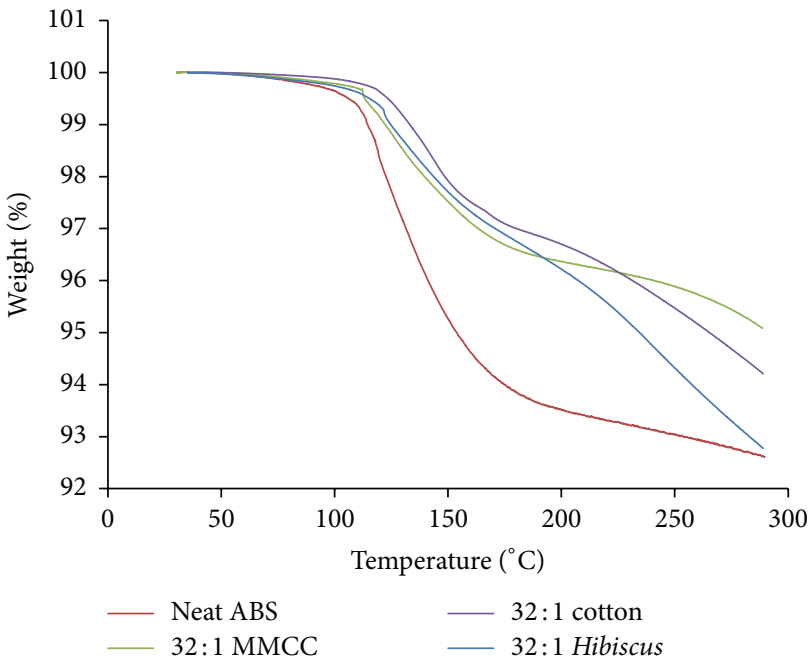

FIGURE 5: TGA percent weight loss curves for purchased and extracted cellulose.

are determined by the acids used, which in turn degrades at varying times and temperatures. It can be observed in Figure 5 and Table 1 that the extracted celluloses begin to degrade at lower temperatures than the MMCC but the rate of decomposition is more favorable for the extractions. Hence, the hydrolysis caused the extracted celluloses to become more thermally resistant at higher temperatures in comparison to the manufactured cellulose. In Table 1 , at $400^{\circ} \mathrm{C}$, MMCC has a residue of $14 \%$, which is lower than that exhibited for its extracted cellulose counterparts. It can be observed that the residues for the extractions are comparable (Hibiscus and cotton, $28.68 \%$ and $32.97 \%$, resp.) but, in comparison to the manufactured, are both approximately doubled in value. It was reported by Kargarzadeh et al. [24] that char percentage increased with acid hydrolysis along with the increase in hydrolysis times. This was said to be ascribed to the increase in the amount of sulfate groups, which acted as flame retardants.

For the extracted celluloses, TGA revealed a slightly higher onset of degradation and maximum decomposition for the Hibiscus in comparison to the cotton, as previously stated. Contrarily, this was not observed for the percent of residue remaining for the extractions. As stated previously, the residues for Hibiscus and cotton extractions are $28.68 \%$ and $32.97 \%$, respectively. Other analysis values may portray a higher stability for the cotton extraction at $400^{\circ} \mathrm{C}$; per the maximum decomposition temperatures, this is not the case.

According to TGA, MMCC is an ideal candidate for filler due to the thermal stability at high temperatures (i.e., onset 


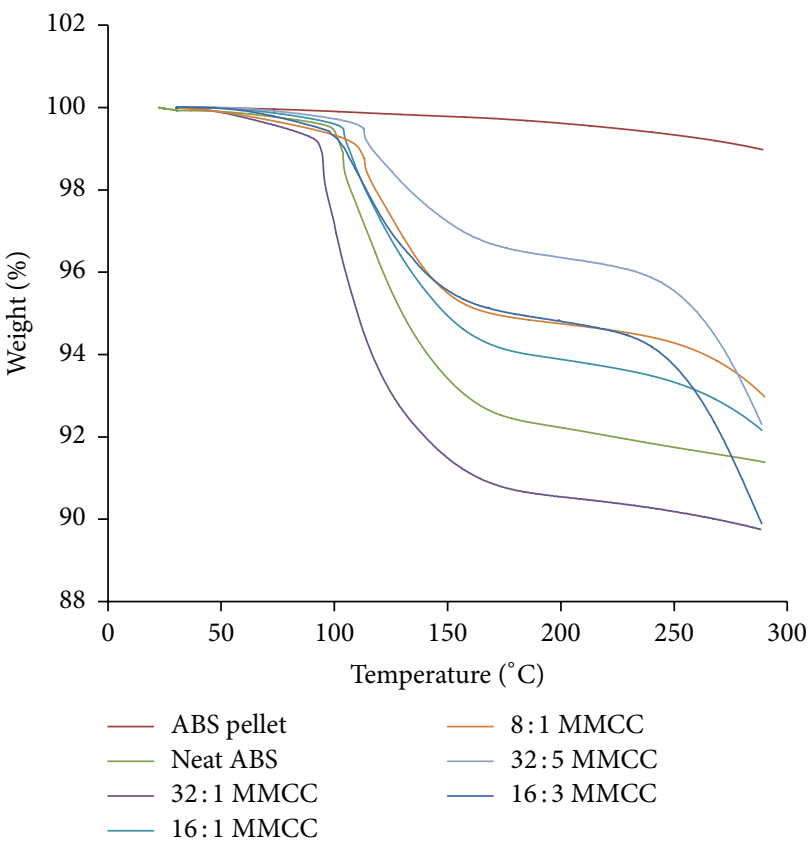

(a)

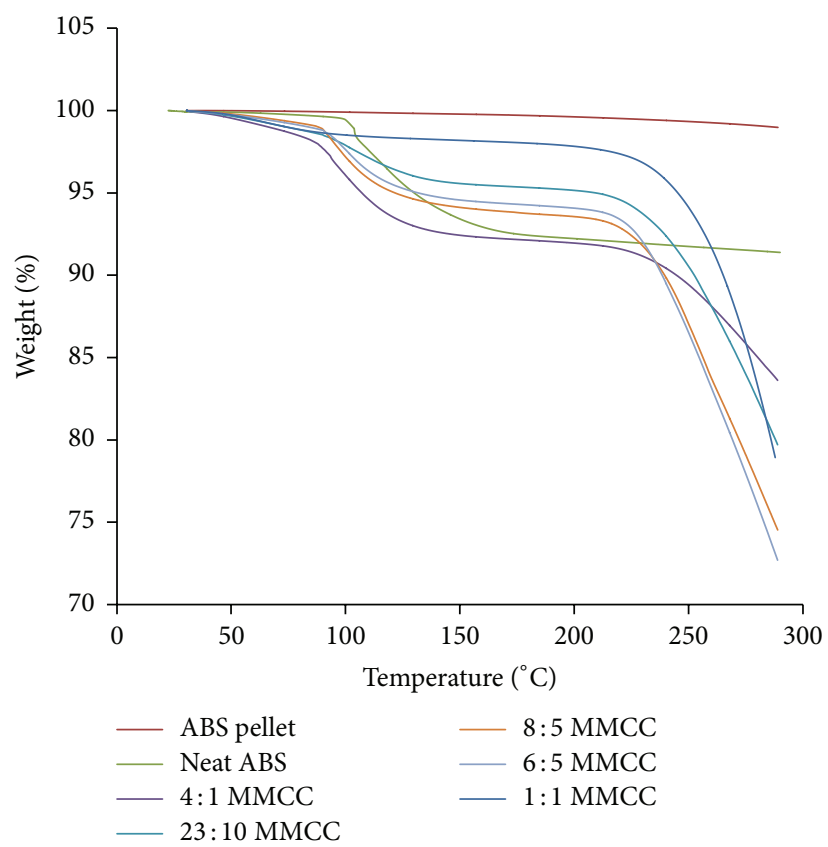

(b)

FIGURE 6: TGA weight loss curves revealing the thermodynamic behavior of neat and MMCC-based ABS plastic composites at various filling ratios (a) and (b).

temperature and maximum decomposition temperature). Onset and maximum decomposition are very important properties when determining thermal stability of materials. Hence, the values presented in Table 1 for MMCC are the determining factors for considering the manufactured cellulose as filler. The $T_{g}$ was not revealed in DSC for the celluloses.

\subsection{Thermal Analysis of Cellulose-Based ABS Composites.} Figures 6(a) and 6(b) show thermal graphs for the cellulose filled at different ratios of ABS to cellulose and neat ABS. Upon initial observation of the thermal graphs featured in Figures 6(a) and 6(b), the neat polymer sample has an onset temperature higher than that of the as-received ABS pellet. Based on this observation, we must note that the method utilized for the fabrication of the neat ABS and cellulosebased ABS composites apparently has a small initial effect on their thermal stability $[18,32]$. In the case of the neat ABS, the slight improvement in thermal stability when compared to the pellet ABS form can be attributed to the decrease in density of dangling bonds of the ABS structure. Nishi et al. [18] reported that molecules of the solvent used in the solvent casting process of ABS can be absorbed by the ABS polymer causing segments of ABS polymer structure with dangling bonds to be attracted to the solvent molecules leading to improvements in its impact strength. In addition, it is proposed in this study that the same weak intermolecular attractions between the absorbed solvent molecules and the dangling bonds of the ABS provide a mechanism for a slight instantaneous improvement in the thermal stability of the ABS composite. That is, due to the increased intermolecular attractions, a slight increase in the thermal energy needed to promote degradation is observed. However, the residue and rate of degradation for the pellet are more favorable characteristics in comparison to the composites. This could be due to, in the neat ABS case, observance of solvent absorption for the ABS composites. Furthermore, the rate of residue and moisture loss (weight loss) increased as the amount of cellulose increased, with decreasing ABS, proving that the ABS moisture absorbance significantly impacts the thermal stability of the composite material (data not shown).

It can be observed that the incorporation of cellulose microcrystals does not significantly change the initial onset of degradation and the maximum decomposition temperature at low concentrations of cellulose fillers added to the ABS matrix when compared to its neat ABS counterpart; however, significant changes in the thermal stability are observed when the concentration of cellulose fillers is increased. A more quantitative view can be observed from Table 2, which consists of a list of tabulated values for the thermal analyses depicted in Figures 6(a) and 6(b). It must be noted that the onset temperature is significantly higher than that of the asreceived pellet in all samples where cellulose has been added. However, upon comparison to neat ABS samples with the cellulose filled composites, the onset temperatures recorded for the higher ratios of ABS to cellulose (e.g., 32:1, 16:1, $8: 1,32: 5$, and $16: 1$ ) exhibit values that are similar to the average onset temperature of $100.90^{\circ} \mathrm{C}$ measured for its neat ABS counterpart. Furthermore, increasing the concentration of cellulose in the ABS matrix (e.g., $4: 1,23: 10,8: 5,6: 5$, and $1: 1)$ exhibits a significant decrease in the onset temperature when compared to the neat ABS. 
TABLE 2: Tabulated DSC and TGA values for neat and cellulose-based ABS composites.

\begin{tabular}{lccccc}
\hline Ratios $($ ABS : cellulose $)$ & $T_{g}\left({ }^{\circ} \mathrm{C}\right)$ & Residue $(\%)$ & Onset $\left({ }^{\circ} \mathrm{C}\right)$ & Rate $\left(\% /{ }^{\circ} \mathrm{C}\right)$ & Max decomp $\left({ }^{\circ} \mathrm{C}\right)$ \\
\hline Pellet & $104.45 \pm 0.22$ & $98.75 \pm 0.08$ & $62.86 \pm 2.97$ & $0.003 \pm 0.001$ & $162.79 \pm 4.33$ \\
Neat & $93.20 \pm 5.07$ & $91.59 \pm 0.33$ & $100.90 \pm 2.48$ & $0.21 \pm 0.02$ & $108.27 \pm 3.21$ \\
$32: 1$ MMCC & $93.02 \pm 6.34$ & $90.37 \pm 1.14$ & $91.90 \pm 3.40$ & $0.22 \pm 0.06$ & $100.16 \pm 4.85$ \\
$16: 1$ MMCC & $91.48 \pm 7.07$ & $90.49 \pm 1.10$ & $97.03 \pm 3.50$ & $0.19 \pm 0.04$ & $104.02 \pm 3.49$ \\
$8: 1$ MMCC & $88.46 \pm 1.99$ & $91.59 \pm 1.01$ & $103.69 \pm 1.88$ & $0.13 \pm 0.04$ & $111.63 \pm 5.69$ \\
$32: 5$ MMCC & $92.10 \pm 6.65$ & $88.33 \pm 0.66$ & $93.87 \pm 1.57$ & $0.18 \pm 0.04$ & $98.67 \pm 1.17$ \\
$16: 3$ MMCC & $93.05 \pm 7.65$ & $87.48 \pm 1.39$ & $97.63 \pm 3.41$ & $0.18 \pm 0.07$ & $103.07 \pm 5.31$ \\
$4: 1$ MMCC & $95.87 \pm 2.64$ & $81.42 \pm 1.26$ & $82.86 \pm 1.47$ & $0.26 \pm 0.07$ & $90.62 \pm 3.41$ \\
$23: 10$ MMCC & $98.07 \pm 1.77$ & $77.86 \pm 0.93$ & $85.33 \pm 2.11$ & $0.29 \pm 0.02$ & $282.74 \pm 1.47$ \\
$8: 5$ MMCC & $98.55 \pm 5.23$ & $73.99 \pm 0.44$ & $87.00 \pm 1.10$ & $0.32 \pm 0.01$ & $281.91 \pm 2.10$ \\
$6: 5$ MMCC & $97.13 \pm 1.12$ & $71.75 \pm 0.59$ & $87.52 \pm 0.50$ & $0.37 \pm 0.01$ & $283.39 \pm 0.69$ \\
$1: 1$ MMCC & $105.67 \pm 0.09$ & $75.06 \pm 1.08$ & $59.21 \pm 6.07$ & $0.50 \pm 0.01$ & $282.29 \pm 1.87$ \\
\hline $32: 1$ cotton & $94.55 \pm 6.59$ & $93.04 \pm 0.90$ & $109.45 \pm 6.99$ & $0.09 \pm 0.02$ & $131.95 \pm 9.43$ \\
$32: 1$ Hibiscus & $98.39 \pm 6.69$ & $92.40 \pm 0.42$ & $116.07 \pm 4.84$ & $0.10 \pm 0.04$ & $124.19 \pm 2.93$ \\
\hline
\end{tabular}

Unlike the onset temperature trend, the complete opposite is observed for the maximum decomposition temperature of the cellulose filled ABS composites. Although the maximum decomposition temperature is similar for both the neat and cellulose filled ABS composites at the lower concentration of MMCC, as the MMCC concentration increases, the thermal stability "maximum decomposition temperature" increases by a two-fold excess. In fact, at a ratio of $1: 1$, ABS : MMCC, respectively, the onset temperature is lower than the neat ABS but the decomposition temperature is maximized. Given that the MMCC exhibits high thermal stability (ca. $333.37^{\circ} \mathrm{C}$ ), it can be extrapolated that the increase in thermal stability here corresponds closely to that of the thermal properties of MMCC shown in Figures 4 and 5 and, more closely, Table 1 . That is, the increased thermal stability here is surely attributed to the larger amount of cellulose inducing a composite effect when trapped in the ABS matrix. Šišáková [33] and Xanthos [34] have reported that the addition of reinforcements along with the stage of mixing and distribution of fillers within the polymer matrix will significantly impact the thermal and mechanical properties. El-Shekeil et al. [35] found that an increase in filler content decreased the thermal stability of kenaf fiber (Hibiscus cannabinus) reinforced thermoplastic polyurethane composites. At lower temperatures, this was said to be attributed to dehydration from cellulose and thermal cleavage of glycosidic linkage, caused by transglycosylation and scission of C$\mathrm{O}$ and $\mathrm{C}-\mathrm{C}$ bonds. At higher temperatures, aromatization, involving dehydration reactions, was said to have caused decomposition. Furthermore, it has been reported by Xue et al. [36] that excessive dosages of additives no longer fulfill their purpose and perform counterproductively.

Sanaeepur et al. [22] reported a $T_{g}$ value of $108.98^{\circ} \mathrm{C}$ for pure ABS which is shown to be comparable to our ABS pellets. In Table 2, it can be observed that the process described in this paper does not significantly reduce the $T_{g}$ value for ABS; in fact, in every composite the $T_{g}$ value is shown to be comparable. In comparison to the pellet, the $1: 1$ ratio is almost identical to $T_{g}$ of the pellet.

Upon incorporation of cellulose fibers from different biomass sources (see Table 2), a significant change in the $T_{g}$ and maximum decomposition temperature is observed. In the case of cellulose fibers extracted from cotton and Hibiscus, $T_{g}$ was very comparable. The maximum decomposition temperature is observed at $131.36^{\circ} \mathrm{C}, 124^{\circ} \mathrm{C}$, and $100.16^{\circ} \mathrm{C}$ for the cotton fiber, Hibiscus fiber, and MMCC composites, respectively. This could be explained in terms of fiber uniformity and dispersion within the polymer matrix [33]. In the case of the extracted cellulose, it is observed that the cellulose crystals are slightly more uniform in shape and size for both of the cotton and Hibiscus fibers; see Figures 3(b) and 3(c). Irregularities in the shape and size of fillers will impact the uniformity of the fiber dispersion within the polymer matrix during mixing. Furthermore, for the composites consisting of extracted celluloses (see Figure 7), they have the greatest thermal characteristics when compared to neat and 32:1 MMCC counterpart samples in Table 2 and Figure 7. The sulfate groups [2] introduced onto the surface of the celluloses extracted from cotton and Hibiscus are the cause behind the improved thermal properties. These sulfate groups are known to produce charged surfaces that stabilize [2] dispersion. The MMCC composites are expected to have poorer dispersion of particles due the lack of hydrolysis being performed. Without hydrolysis or another form of chemical functionalization, cellulose naturally undergoes hydrogen bonding due to the three hydroxyl groups located on each glucose monomer, causing nonuniform dispersion [25]. As chemical functionalization has the ability to disrupt the strong hydrogen bonding behavior [25], the extracted celluloses have the ability to disperse more uniformly, hence causing a more thermally stable composite.

3.5. Thermomechanical Analysis of ABS Composites. Figure 8 shows the coefficient of thermal expansion (CTE) trend 


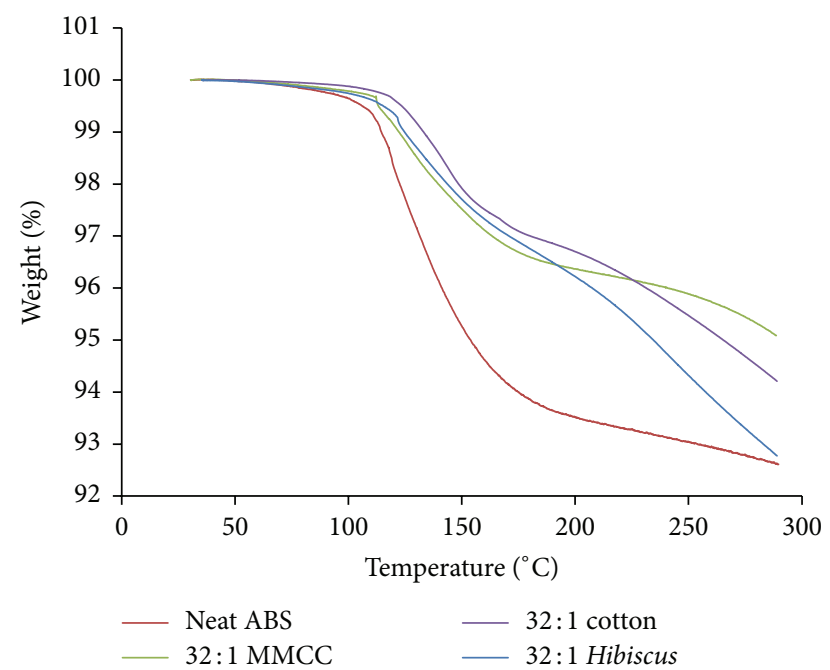

FIGURE 7: TGA weight loss curves revealing the thermodynamic behavior of ABS plastic composites as a function of the ratio of cellulose additive (purchased or extracted).

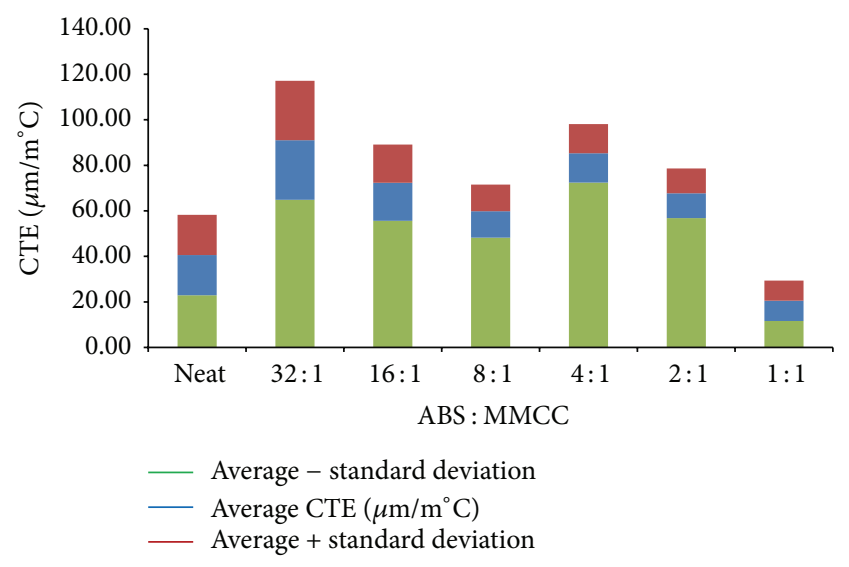

FIGURE 8: TMA bar graphs revealing the thermomechanical behavior of neat and cellulose-based ABS plastic composites.

observed for the ABS composites filled with MMCC. The initial CTE of the neat ABS is observed at around $20 \mu \mathrm{m} / \mathrm{m}^{\circ} \mathrm{C}$. Upon the addition of $3 \%$ MMCC, the CTE increases dramatically. Further increases in the amount of MMCC dispersed within the polymer matrix yield a decrease in the measured CTE values. However, after the addition of 25\% MMCC fillers (i.e., $4: 1$ ratio), a dramatic increase in the CTE values is observed with a continued decrease as the amount of cellulose fillers is increased within the polymer matrix. It is well known that the shape and the dispersion of fillers in the polymer matrix significantly impact its effect on the measured CTE of its polymer host composite. DeSarkar and coworkers [37] reported that upon the addition of various inorganic fillers a dramatic change was observed for the polycarbonate matrix and effectiveness of the impact of the fillers on the measured CTE values of the polymer matrix was dependent upon filler type and degree of dispersion. That is, better dispersion of the fillers within the polymer matrix allowed for greater interaction with the matrix, thereby decreasing the CTE value. In this study, mechanical methods were not used to mix the cellulose-based ABS composites, but rather a rudimentary method was used such as wooden stir sticks. The uniformity of the dispersion during this process is unknown and questionable in its dispersion homogeneity. Thus, we speculate that the dispersion of the MMCC within the ABS matrix governs the CTE trends observed for the cellulosebased composites. (Note that investigations to determine the nature of the dispensability of our cellulose fillers within ABS polymer network via mixing method used here are currently in progress.) Furthermore, the CTE value improves significantly for the $1: 1$ ratio of $\mathrm{ABS}$ to cellulose when compared to the neat ABS. Cellulose and wood pulp has been found to have a low CTE, as well as having the capabilities of reducing the CTE of composites [38-40]. The CTE for the cellulose in this paper needs to be determined for a conclusive answer but the large values and variations in the composites observed can be attributed to the possible heterogeneous mixture of the MMCC within the matrix. Additionally, the presence of moisture and thermal stresses play a vital role in the properties of these composites.

\section{Conclusions}

In this paper, we have found that extraction and drying procedures of cellulose have a dramatic effect on dispersion and thermal stability of polymer matrices. We have also discovered that polymer dissolution is a very useful technique for the improvement of cellulose filled ABS composites. With cellulose having an effect on the properties of ABS, $T_{g}$ was not dramatically lowered, which is a great quality for manufacturing. Although the CTE does not have a distinct trend, the average minus the standard deviation (STD) for all samples falls below that whcih is reported.

\section{Competing Interests}

The authors declare that they have no competing interests.

\section{Acknowledgments}

The authors gratefully acknowledge the National Science Foundation under Grants nos. NSF EPS-1158862, NSF HRD1137681, and NSF IGERT on Sustainable Electronics DGE1144843 for support of this research. The Department of Materials Science and Engineering and Department of Chemistry and Chemistry Center for Synthesis and Characterization are recognized.

\section{References}

[1] I. Vroman and L. Tighzert, "Biodegradable polymers," Materials, vol. 2, no. 2, pp. 307-344, 2009.

[2] R. J. Moon, A. Martini, J. Nairn, J. Simonsen, and J. Youngblood, "Cellulose nanomaterials review: structure, properties and nanocomposites," Chemical Society Reviews, vol. 40, no. 7, pp. 3941-3994, 2011. 
[3] S. Baruah, "Biodegradable polymer: the promises and the problems," Science and Culture, vol. 77, pp. 466-470, 2011.

[4] G. Siaueira, J. Bras, and A. Dufresne, "Cellulose whiskers versus microfibrils: influence of the nature of the nanoparticle and its surface functionalization on the thermal and mechanical properties of nanocomposites," Biomacromolecules, vol. 10, no. 2, pp. 425-432, 2009.

[5] H. Qi, J. Cai, L. Zhang, and S. Kuga, "Properties of films composed of cellulose nanowhiskers and a cellulose matrix regenerated from alkali/urea solution," Biomacromolecules, vol. 10, no. 6, pp. 1597-1602, 2009.

[6] W. Gindl and J. Keckes, "All-cellulose nanocomposite," Polymer, vol. 46, no. 23, pp. 10221-10225, 2005.

[7] M. Yousefi, F. Gholamian, D. Ghanbari, and M. SalavatiNiasari, "Polymeric nanocomposite materials: preparation and characterization of star-shaped $\mathrm{PbS}$ nanocrystals and their influence on the thermal stability of acrylonitrile-butadienestyrene (ABS) copolymer," Polyhedron, vol. 30, no. 6, pp. 10551060, 2011.

[8] D. Bondeson and K. Oksman, "Polylactic acid/cellulose whisker nanocomposites modified by polyvinyl alcohol," Composites Part A: Applied Science and Manufacturing, vol. 38, no. 12, pp. 2486-2492, 2007.

[9] E. de Morais Teixeira, A. C. Corrêa, A. Manzoli, F. de Lima Leite, C. de Ribeiro Oliveira, and L. H. C. Mattoso, "Cellulose nanofibers from white and naturally colored cotton fibers," Cellulose, vol. 17, no. 3, pp. 595-606, 2010.

[10] B. Wang, M. Sain, and K. Oksman, "Study of structural morphology of hemp fiber from the micro to the nanoscale," Applied Composite Materials, vol. 14, no. 2, pp. 89-103, 2007.

[11] L. Suryanegara, A. N. Nakagaito, and H. Yano, "The effect of crystallization of PLA on the thermal and mechanical properties of microfibrillated cellulose-reinforced PLA composites," Composites Science and Technology, vol. 69, no. 7-8, pp. 1187$1192,2009$.

[12] J. Lu, P. Askeland, and L. T. Drzal, "Surface modification of microfibrillated cellulose for epoxy composite applications," Polymer, vol. 49, no. 5, pp. 1285-1296, 2008.

[13] K. A. Iyer, G. T. Schueneman, and J. M. Torkelson, "Cellulose nanocrystal/polyolefin biocomposites prepared by solid-state shear pulverization: superior dispersion leading to synergistic property enhancements," Polymer, vol. 56, pp. 464-475, 2015.

[14] K. Oksman, Y. Aitomäki, A. P. Mathew et al., "Review of the recent developments in cellulose nanocomposite processing," Composites Part A: Applied Science and Manufacturing, vol. 83, pp. 2-18, 2016.

[15] K. A. Iyer, A. M. Flores, and J. M. Torkelson, "Comparison of polyolefin biocomposites prepared with waste cardboard, microcrystalline cellulose, and cellulose nanocrystals via solidstate shear pulverization," Polymer, vol. 75, pp. 78-87, 2015.

[16] K. Vanhatalo, N. Maximova, A. Perander, L. Johansson, E. Haimi, and O. Dahl, "Comparison of conventional and ligninrich microcrystalline cellulose," BioResources, vol. 11, no. 2, pp. 4037-4054, 2016.

[17] C. J. Huntley, K. D. Crews, M. A. Abdalla, A. E. Russell, and M. L. Curry, "Influence of strong acid hydrolysis processing on the thermal stability and crystallinity of cellulose isolated from wheat straw," International Journal of Chemical Engineering, vol. 2015, Article ID 658163, 11 pages, 2015.

[18] Y. Nishi, N. Kunikyo, M. Kanda, L. Lebrun, and D. Guyomar, "Impact value of high electric conductive ABS composites with copper powder dispersion prepared by solution-cast method," Materials Transactions, vol. 51, no. 1, pp. 165-170, 2010.

[19] M. A. Hubbe, O. J. Rojas, L. A. Lucian, and M. Sain, "Cellulosic nanocomposites: a review," BioResources, vol. 3, no. 3, pp. 929$980,2008$.

[20] J. V. Rutkowski and B. C. Levin, "Acrylonitrile-ButadieneStyrene copolymers (ABS): pyrolysis and combustion products and their toxicity - a review of the literature," Fire and Materials, vol. 10, no. 3-4, pp. 93-105, 1986.

[21] D. A. Contos, M. W. Holdren, D. L. Smith, R. C. Brooke, V. L. Rhodes, and M. L. Rainey, "Sampling and analysis of volatile organic compounds evolved during thermal processing of acrylonitrile butadiene styrene composite resins," Journal of the Air \& Waste Management Association, vol. 45, no. 9, pp. 686694, 1995.

[22] H. Sanaeepur, A. E. Amooghin, A. Moghadassi, and A. Kargari, "Preparation and characterization of acrylonitrile-butadienestyrene/poly(vinyl acetate) membrane for $\mathrm{CO}_{2}$ removal," Separation and Purification Technology, vol. 80, no. 3, pp. 499-508, 2011.

[23] E. N. J. Ford, S. K. Mendon, S. F. Thames, and J. W. Rawlins, "X-ray diffraction of cotton treated with neutralized vegetable oil-based macromolecular crosslinkers," Journal of Engineered Fibers and Fabrics, vol. 5, no. 1, pp. 10-20, 2010.

[24] H. Kargarzadeh, I. Ahmad, I. Abdullah, A. Dufresne, S. Y. Zainudin, and R. M. Sheltami, "Effects of hydrolysis conditions on the morphology, crystallinity, and thermal stability of cellulose nanocrystals extracted from kenaf bast fibers," Cellulose, vol. 19, no. 3, pp. 855-866, 2012.

[25] C. J. Huntley, K. D. Crews, and M. L. Curry, "Chemical functionalization and characterization of cellulose extracted from wheat straw using acid hydrolysis methodologies," International Journal of Polymer Science, vol. 2015, Article ID 293981, 9 pages, 2015.

[26] A. C. O'Sullivan, "Cellulose: the structure slowly unravels," Cellulose, vol. 4, no. 3, pp. 173-207, 1997.

[27] A. K. Bledzki and J. Gassan, "Composites reinforced with cellulose based fibres," Progress in Polymer Science, vol. 24, no. 2, pp. 221-274, 1999.

[28] S. Park, J. O. Baker, M. E. Himmel, P. A. Parilla, and D. K. Johnson, "Cellulose crystallinity index: measurement techniques and their impact on interpreting cellulase performance," Biotechnology for Biofuels, vol. 3, article 10, 2010.

[29] J. K. Pandey, A. N. Nakagaito, and H. Takagi, "Fabrication and applications of cellulose nanoparticle-based polymer composites," Polymer Engineering \& Science, vol. 53, no. 1, pp. 1-8, 2013.

[30] E. Sinha and S. K. Rout, "Influence of fibre-surface treatment on structural, thermal and mechanical properties of jute fibre and its composite," Bulletin of Materials Science, vol. 32, no. 1, pp. 65-76, 2009.

[31] Office of Water EPA, The Pulp and Paper Industry, the Pulping Process, and Pollutant Releases to the Environment, 1997.

[32] Y. Nishi, Y. Ebihara, N. Kunikyoh et al., "High electric conductive PMMA composites without impact value decay by dispersion of copper powder," Materials Transactions, vol. 51, no. 8, pp. 1437-1442, 2010.

[33] J. Šišáková, "Polymer materials and its properties," in New Trends in Technologies: Devices, Computer, Communication and Industrial Systems, M. J. Er, Ed., chapter 2, pp. 15-42, InTech, Rijeka, Croatia, 2010.

[34] M. Xanthos, "Polymers and polymer composites," in Functional Fillers for Plastics, M. Xanthos, Ed., pp. 1-18, 2010. 
[35] Y. A. El-Shekeil, S. M. Sapuan, K. Abdan, and E. S. Zainudin, "Influence of fiber content on the mechanical and thermal properties of Kenaf fiber reinforced thermoplastic polyurethane composites," Materials \& Design, vol. 40, pp. 299-303, 2012.

[36] M.-L. Xue, Y.-L. Yu, H. H. Chuah, J. M. Rhee, N. H. Kim, and J. H. Lee, "Miscibility and compatibilization of poly(trimethylene terephthalate)/acrylonitrile-butadiene-styrene blends," European Polymer Journal, vol. 43, no. 9, pp. 3826-3837, 2007.

[37] M. DeSarkar, P. Senthilkumar, S. Franklin, and G. Chatterjee, "Effect of particulate fillers on thermal expansions and other critical performances of polycarbonate-based compositions," Journal of Applied Polymer Science, vol. 124, no. 1, pp. 215-226, 2012.

[38] T. Nishino, I. Matsuda, and K. Hirao, "All-cellulose composite," Macromolecules, vol. 37, no. 20, pp. 7683-7687, 2004.

[39] Y. Okahisa, A. Yoshida, S. Miyaguchi, and H. Yano, "Optically transparent wood-cellulose nanocomposite as a base substrate for flexible organic light-emitting diode displays," Composites Science and Technology, vol. 69, no. 11-12, pp. 1958-1961, 2009.

[40] S. Iwamoto, K. Abe, and H. Yano, "The effect of hemicelluloses on wood pulp nanofibrillation and nanofiber network characteristics," Biomacromolecules, vol. 9, no. 3, pp. 1022-1026, 2008. 

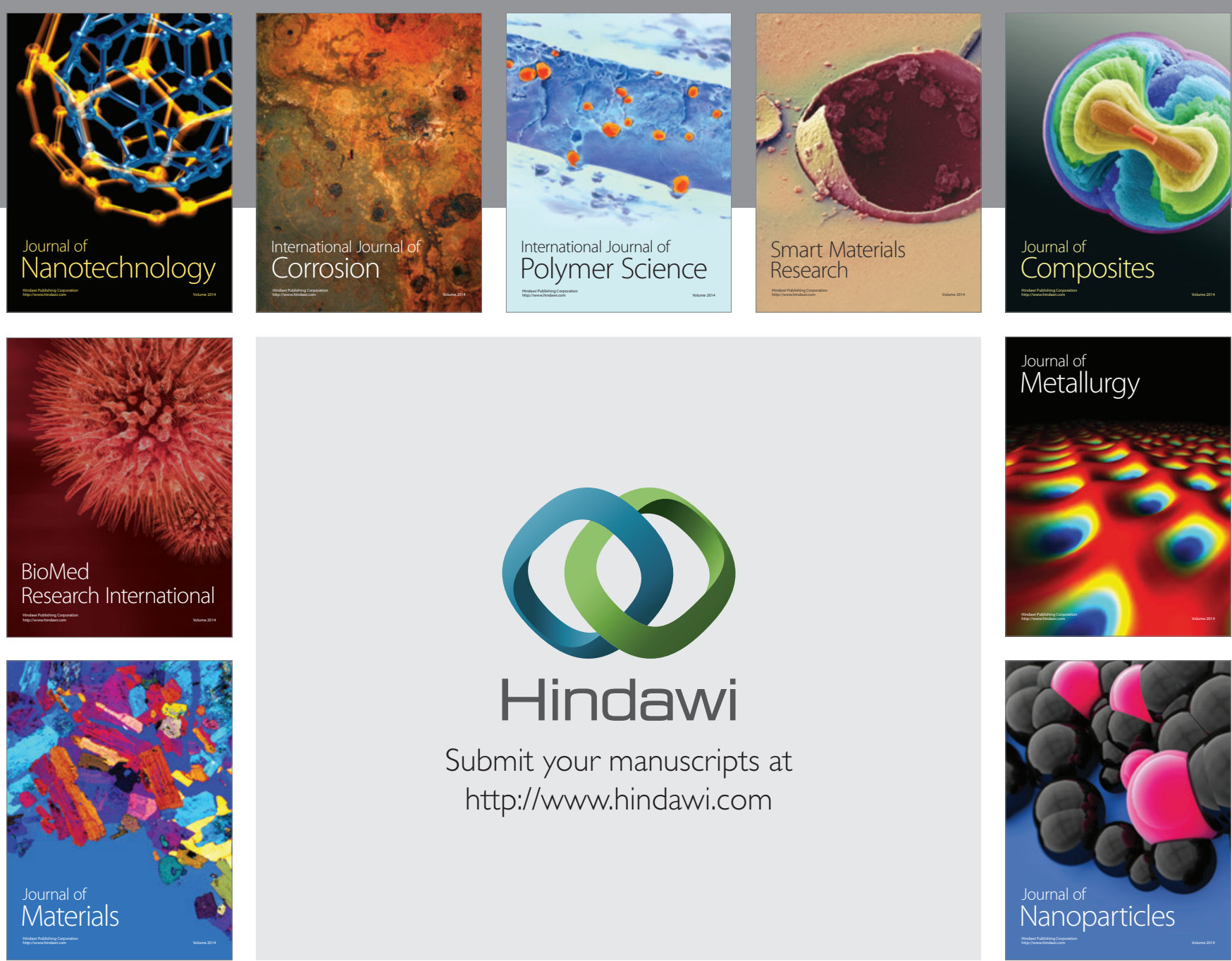

\section{Hindawi}

Submit your manuscripts at

http://www.hindawi.com

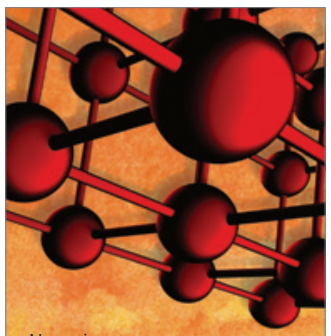

Materials Science and Engineering
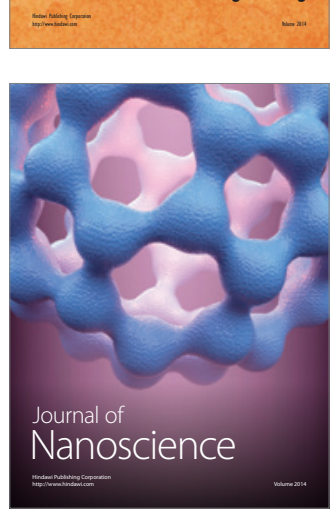
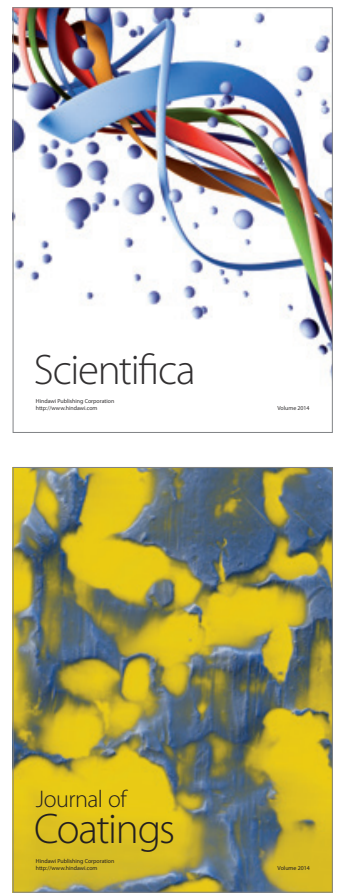
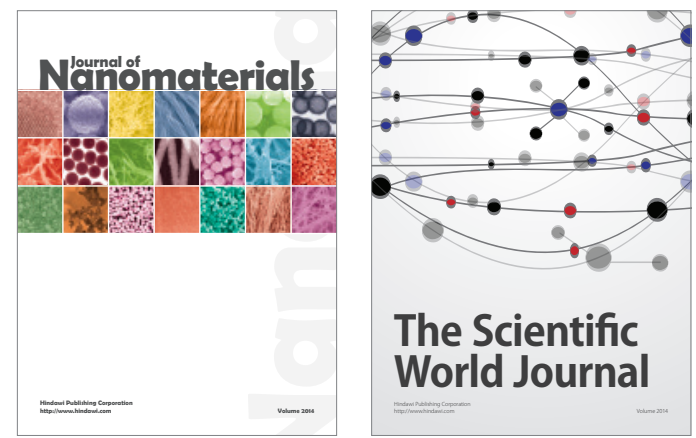

The Scientific World Journal
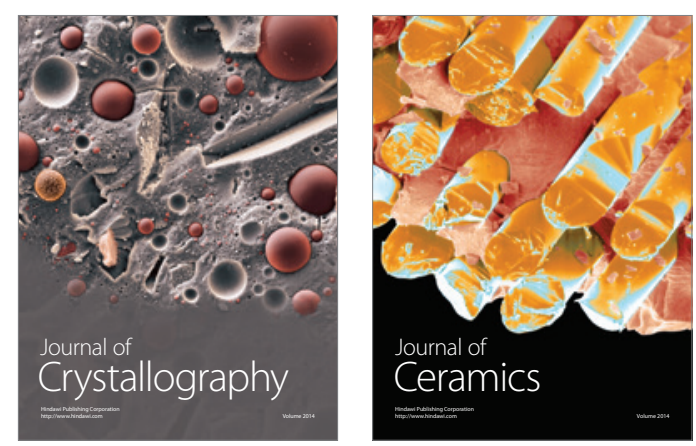
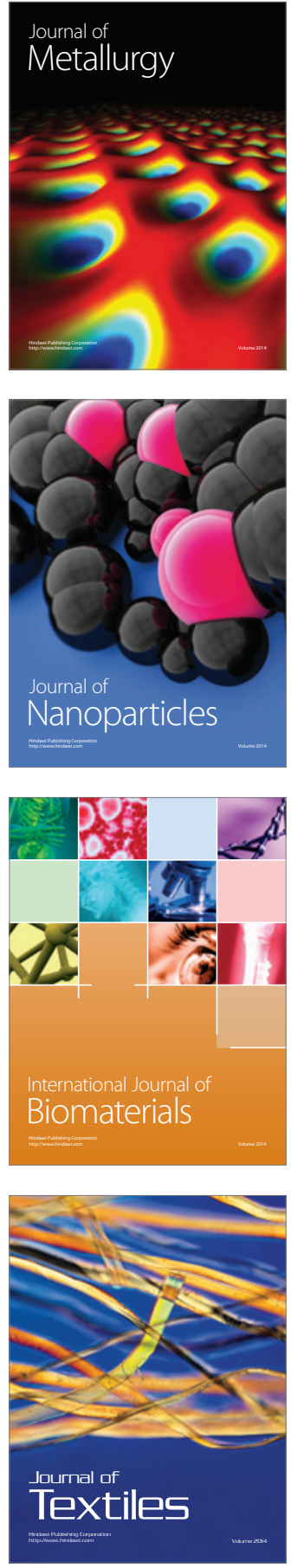